\title{
Design and Implementation of Augmented Reality Environment for Complex Anatomy Training: Inguinal Canal Case Study
}

\author{
S. Sakellariou ${ }^{1}$, B.M. Ward ${ }^{2}$, Vassilis Charissis ${ }^{3}$, D. Chanock $^{4}$, and P. Anderson ${ }^{3}$ \\ ${ }^{1}$ Aberdeen Royal Infirmary \\ Acute Medicine, Aberdeen, UK \\ \{s.sakellariou@nhs.net\} \\ University of Edinburgh, UK \\ ${ }^{3}$ University of Glasgow/ Glasgow School of Art, Digital Design Studio, \\ 10 Dumbreck Road, G41 5BW, Glasgow, UK \\ \{v.charissis@gsa.ac.uk\} \\ ${ }^{4}$ Ayr Hospital \\ Department of Radiology, Ayr, UK
}

\begin{abstract}
Adhering to contemporary requirements for reduction of cadaveric training of medical trainees we have developed a prototype augmented reality environment which investigates complex anatomical sections. A human 3D model has been implemented in order to facilitate educational tactics presented in a Virtual Reality (VR) environment. Opting for a sophisticated approach of interaction, the interface elements are based on simplified visual representation of real anatomical elements, and can be operated through haptic devices and surround auditory cues. This paper discusses the challenges involved in the development process of the augmented reality environment, and the HCI design, introduces the visual components of the interface and presents the outcome of a preliminary evaluation of the proposed VR training method on a group of twelve medical doctors. The paper concludes with a tentative plan of future work which aims to expand the context and interactivity of the system so as to enable the trainees to rehearse surgical methods in a simulated VR environment.
\end{abstract}

Keywords: Virtual Reality, Haptics, HCI, Inguinal Canal, Medical Training

\section{Introduction}

The propagation of augmented reality representations and advanced humancomputer interaction techniques presented momentous advances in the accretion of knowledge with regards to complex human-body regions $[1,2]$. To this point it has to be noted that the knowledge acquisition and ultimately the understanding of such motley three-dimensional subjects typically entails a strong grasp of the 3D anatomy 
to which it relates $[3,4]$. This is compounded when procedural techniques and specialist anatomy are effectively taught simultaneously. It has been shown however, that anatomy learning can be augmented by the use of high resolution 3D models and intuitive human-computer interaction $[5,6,7,8]$. To this end, we have endeavoured in the investigation of various case studies which incorporate a high level of anatomical complexity which would demand for a highly versatile interface design. One such study, the inguinal canal region, was of particular interest, as the different abdominal layers forming the canal are exceptionally difficult to comprehend spatially.

Adhering to the aforementioned observations, a detailed 3D representation of the inguinal canal and the extended abdominal area was developed through the data acquisition by CT scans, laser scanning and MRI scans, under the consultation of the involved medical doctors. Utilising the departmental VR facilities we developed a custom user-interface which could facilitate a visual, haptic and auditory navigation throught the physical layers of the 3D model. The particular system entails real-time visualisation, gesture interaction with tactile feedback (CyberTouch $^{\mathrm{TM}}$ sensored glove) and 3D sound developed around a Fakespace Immersive Workbench.

A comparative study between existing teaching material and the proposed VR training method was deemed necessary in order to identify the potential benefits, if any, of the proposed system.

Overall this study presents a concise but enlightening assessment on the involvement of a VR learning environment and advanced user-interfaces for medical training. The paper explores the empirical evidence regarding 3D visualisation and the enhancement of spatial learning and describes the integration of robust anatomical modeling techniques, intuitive human-computer interfaces (HCI) and current educational theory. The paper concludes with a succinct discussion of the deliverable feedback and suggestions from both trainee and consultant doctors and offers a provisional plan for further development.

\section{Inguinal Canal Case Study}

The inguinal canal region is broadly perceived in medical education as an explicitly arduous section of the human body; hence the medical doctors involved in this project suggested the particular section for further investigation through an augmented reality environment where physical constrains can be shifted.

The main difficulty towards the understanding of the special relation in -between the different elements occurs predominantly due to the complexity of the layered structure of muscles and fascias involved in the creation of the canal which forms the passage for the spermatic cord. Elaborating in the aforementioned observation it is evident that the male inguinal canal is the route by which the vas deferens and testicular neurovascular bundle passes through the musculature of the anterior abdominal wall. While the functionality of this system could be characterised as trivial in the female version, yet it poses a significant danger, as an area of potential flaws, in both sexes.

These flaws can be encountered typically in the form of three types of hernias which can appear at, or close to the inguinal region. Consequently these hernias can 
be named after their positioning attributes as direct or indirect inguinal and femoral hernias.

Contemporary educational methods for teaching complex anatomical regions are considered inadequate [1] as they typically lack the depiction of a 3D spatial issue in a three dimensional manner. As such the majority of explanatory illustrations are diagrammatic, 2D representations of pre-determined angles of depiction.

Hence it is impossible for the trainee to investigate in depth the layered structures, their spatial relations and visit these complex structures from different angles which might enlighten their perception and understanding. Interestingly the explanatory text and the visual representations of the inguinal canal area appearing in a number of manuscripts are inadequate to convey the complexity and the volume of the information related to this region as presented in Figure 1. In contrary the text describes the position of each of the involved structures in explicit detail, convoluting even further the structural hierarchy of the region.
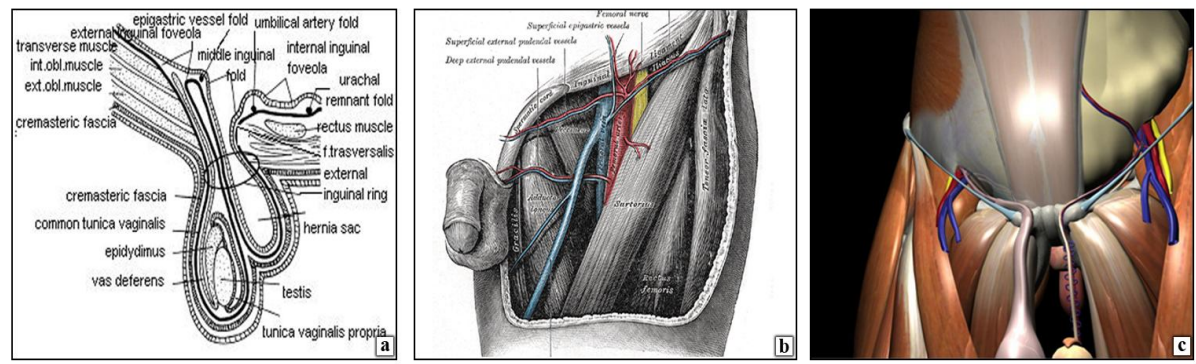

Fig. 1. Samples of teaching illustrations of the Inguinal Canal: (a) Simple 2D diagram,

(b) More detailed 2D depiction, and (c) Screenshot from 3D model

Notably the 2D representations were intended to be used as preparatory means of information to cadaveric dissections and demonstrations which can effectively present the three-dimensional relationships of the canal walls and openings.

However, the shortage of cadaveric material presented a significant issue in this training method which was inevitably reduced to the $2 \mathrm{D}$ representations as the main mean of teaching such regions. In the face of this major concern the only prominent method left to explore, was the digital reconstruction of the human body which offers a better real-life representation as well as a number of tools not applicable to a cadaver (i.e. infinite viewpoints, real-time highlights, colour coding etc.). Furthermore the indestructible ability of the digitised data offers infinite manipulation methods, minimum storage and maintenance requirements.

\section{Development of 3D Dataset}

Mindful of the aforementioned medical training issues, we opted initially for a realistic, yet stylised 3D representation of the human body, which would offer an uncluttered view (i.e. simplification of vein artery and nerves' routes) of the organs under investigation. The primary target of the evaluation was to highlight the spatial 
relation between different layers, organs and structures of the body. In order to achieve an adequate detail and precision to the final result we opted for a multidisciplinary group formed by specialists which included 3D visualisation experts, surgeons, anatomists, radiologists, medical educators, programmers, HCI scientists, and Human-Factor engineers. The derived high fidelity 3D representation of the inguinal canal was focused to compliment an existing activity based curriculum.

Adhering to the above objective we have developed a highly detailed 3D model of the inguinal canal region with particular emphasis in enhancing learning of human anatomy for trainee doctors. While the complexity of the human data was simplified we maintained a significant amount of details that were absolutely relevant to a surgical operation in that region and to the proper depiction of the different pathological cases. This exemplifies the importance of this collaborative methodology, and indeed the difference between taught and surgical operative anatomy.

Notably, each opening (the deep and superficial inguinal rings) was developed in order to be easily visible and seen to be "protected" by two of the muscle layers. The muscles and their aponeuroses were clearly defined and two of them (internal oblique and transversus abdominis) could be seen arching over the canal to form its roof and then its posterior wall (conjoint tendon) as depicted in Figure 2.

Particular emphasis was given to the detailed representation of relations between musculoskeletal parts and fascia layers. Furthermore, all the important surgical landmarks were visually clarified to the user. The final 3D model was in turn customised for stereoscopic viewing (Virtual reality representation) and haptic manipulation in an augmented reality facility.

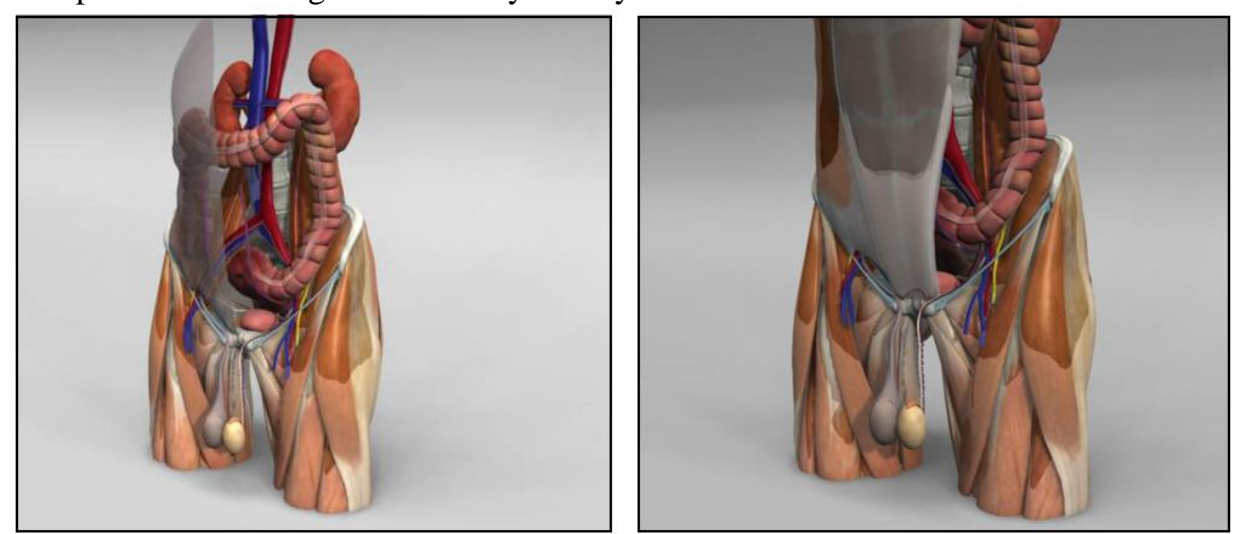

Fig. 2. (a) Real-time transparency change and layer appearance in the $3 D$ Inguinal Canal, (b) Demonstration of different layers appearance and close-up investigation. 


\section{Development of HCI for Medical Teaching}

The purpose of developing such advanced VR system was not to exemplify any technological breakthroughs but to enhance the learning process in a very demanding profession. Hence our endeavour had as a main objective to assist this process by meaningful information provided in a simplistic manner. To this point we have to clarify that the proposed VR system did not aim to substitute the existing teaching methods; in contrary it was designed in conjunction to the existing curriculum so as to compliment the existing practices, yet to accelerate the learning progression and mentally imprint the crucial information.

To this end the interface development particularly aimed to depict meaningful information that could enhance the learning process of the trainee doctors in an augmented reality environment. Hence, our focusing point was the interface functionalities, which will enable the trainees to mentally perceive the threedimensional structure of the human body and navigate through, discovering and understanding convoluted information with regard to complex anatomical regions, such as the inguinal canal.

These interface components present fresh opportunities for the portrayal of information using symbolic and alphanumeric representations and feature an infinite selection of viewing positions than was previously impossible in the cadaver. Our attempt to directly apply publicly accepted interface components to the medical training environment was a challenging process, as the icons had to be designed in accordance to the requirements of each section. To this end the icons were showing miniature representations of the layers as illustrated in the upper toolbar of Figure 3. This was crucial in order to allow the doctors control of key muscular layers that obstruct ready access during hernia procedures thus allowing unparalleled investigative freedom.

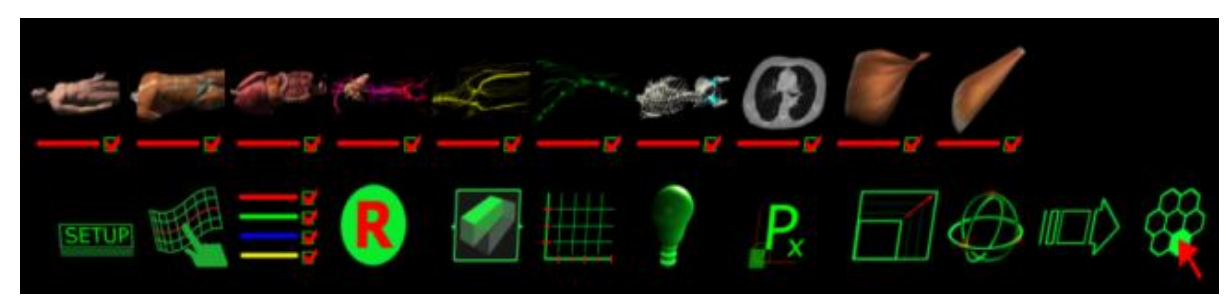

Fig. 3. Augmented Reality HCI: human layers (top), default actions (bottom)

Colour coding was also employed wherever required to aid the understanding of different tissues and functionalities. Notably the development of the 3D model, the VR environment and the proposed HCI aimed to lay the ground for primary laparoscopic surgical rehearsal. 


\section{Visualisation Requirements}

The requirements for visualising and implementing the functionalities of the aforementioned interface were based on hardware equipment existing in the VR facility of Digital Design Studio. The system is designed based on a Fakespace Immersive Workbench and entails real-time visualisation, gesture interaction with tactile feedback (CyberTouch ${ }^{\mathrm{TM}}$ sensored glove) and 3D sound as depicted in figure 4 (a, b). Its semi-immersive design is particularly valuable in assisting with small group tutorial requirements.

The physical dimensions and shape of the table offered intriguingly similar position and dimensions to an operation table or an examination bed. For this reason the existing VR and haptic technology was repositioned in order to enhance this feeling, replicating as much as possible the position of a cadaveric examination as depicted in Figure 4.
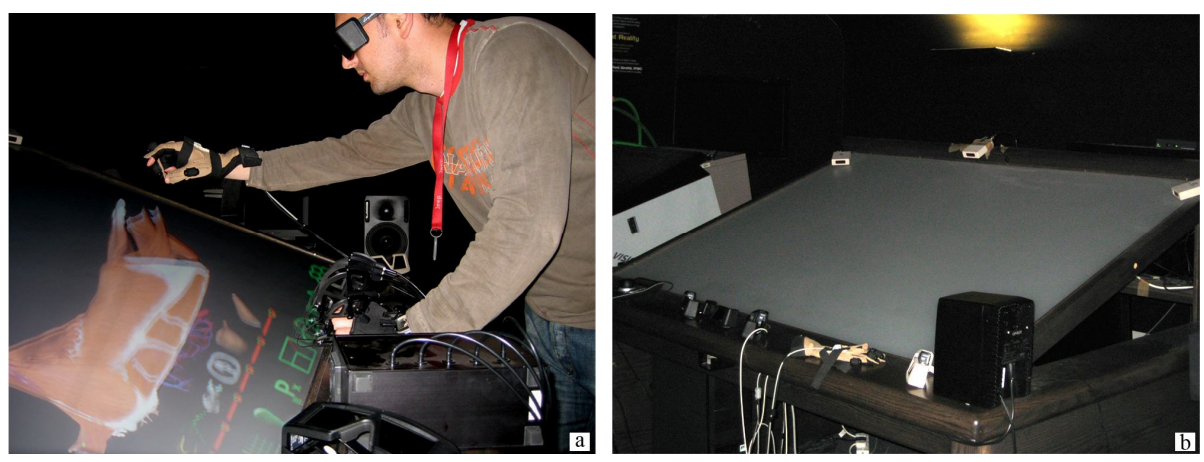

Fig. 4. (a) Haptic interface demonstration for the manipulation of the VR human section (b) The Fakespace Immersive Workbench with the CyberTouch ${ }^{\mathrm{TM}}$ sensored glove.

The stereoscopic visualisation is provided by CrystalEyes shutter glasses, which could provide the trainee and the trainer to have an overall view of the table but also of the surrounding space and users around the table. However only one pair of glasses could be tracked by the table-sensors (typically the person using the haptic glove); therefore the rest of the viewers should be positioned as close as possible to that operating user in order to avoid visual distortion.

The "physical" interaction between user and the 3D model is achieved with the use of a tracked CyberTouch ${ }^{\mathrm{TM}}$ sensored glove with vibro-tactile stimulators on the fingers and palm. This glove can sense the bend and relative position of the fingers and thumb, allowing interaction via gesture; combined with tracking it can sense the hand's position in space, allowing the user to explore and manipulate the digital model directly in 3D space.

Finally 3D spatialised speakers provided the auditory cues. The speakers' network offered sound effects, which signalise a variety of different actions. The depth perception is extensively investigated with sound moves in space appropriate to the manipulation of the model. 


\section{Experiment Rationale}

Subsequently a user-trial experiment was designed in an activity-based curriculum, in order to evaluate the responses of twelve medical trainees. These users were randomly selected to participate in this first phase of the experiment which involved manipulation of the 3D model and identification of each components starting from the pelvis and building up to the skin layer.

The evaluation aimed to contrast the potential benefits and pitfalls of a VR learning environment against the traditional anatomy teaching techniques. Feedback from laparoscopic surgeons was also derived in order to contrast it with the expectations and results of the trainees and their performance using the VR system. Notably the evaluation process involved trainee and fully trained doctors (consultant level) in order to cover both ends of the spectrum.

Their feedback and suggestions formed an initial appraisal of the benefits of the system but also highlighted its potential shortcomings. In general the system was praised for its efficiency in presenting complex 3D data in a comprehensive manner for the user, by circumventing the limitations of the traditional teaching tools. Finally the aforementioned feedback formed our tentative plan for future work which includes further development of the 3D model details and an extensive VR interface to include practical pre-operative surgical rehearsal for core surgical procedures.

The performance of the trainees was measured through a series of pre and post teaching tools as presented below. A usability questionnaire was employed to capture their thoughts and feedback with regards to these two diverse teaching techniques.

1) Pre assess Likert (demographics etc)

2) The pre-assessment quiz (10mins)

3) 5 minutes with the Inguinal Canal activator sheet

4) Intervention (15mins tutorial - focus on the structures on the CT)

5) Post assessment (Inguinal Canal spot test)

6) Usability questionnaire (no limit)

In this paper we are focusing our analysis on the pre and post-assessment results derived by Likert-scale questionnaires which can be indicative of the proposed systems positive aspects as well as the potential arising issues.

\section{Results \& Discussion}

Analysis of the users' pre Likert questionnaires established that the two subgroups were comparable in respect to the following variables: stage of training (all being house officers), previous anatomy training (all being graduates of UK medical schools), exposure to pro-sections and dissections teaching methods (7-11 months), IT literacy, surgical hands-on experience (4-8 months) and focused anatomy training. None has had focused prior training on the inguinal canal region and none had yet attempted the surgical postgraduate exam.

The pre-assess Likert study also explored the users' views on current anatomy teaching and their learning behaviours on interpreting 3D data and complex regional anatomy for clinical use. Their responses were very similar, with no significant 
differences in p-values (t-Test: two sample assuming equal variance) in any of the attitude determining questions $(\mathrm{p}>0.05)$ between the subgroups. Notably, the trainees uniformly expressed the view that current anatomy teaching in undergraduate level and during clinical years is fragmented, limited and lacking in depth, whilst the teaching methods were described as time consuming and non-engaging.

The majority of users found it difficult to construct a mental 3D map of the human anatomy from studying 2D models and thus application of their anatomy knowledge in a clinical interpretation scenario was graded as inadequate for their clinical needs. $80 \%$ of users strongly agreed that further enhancement of their anatomy training will aid their clinical practice with the remaining agreeing with the aforementioned, but not as strongly. Access to 3D anatomical models for training needs was graded as limited and difficult by all users and they all agreed that anatomy is a hard subject to learn from books.

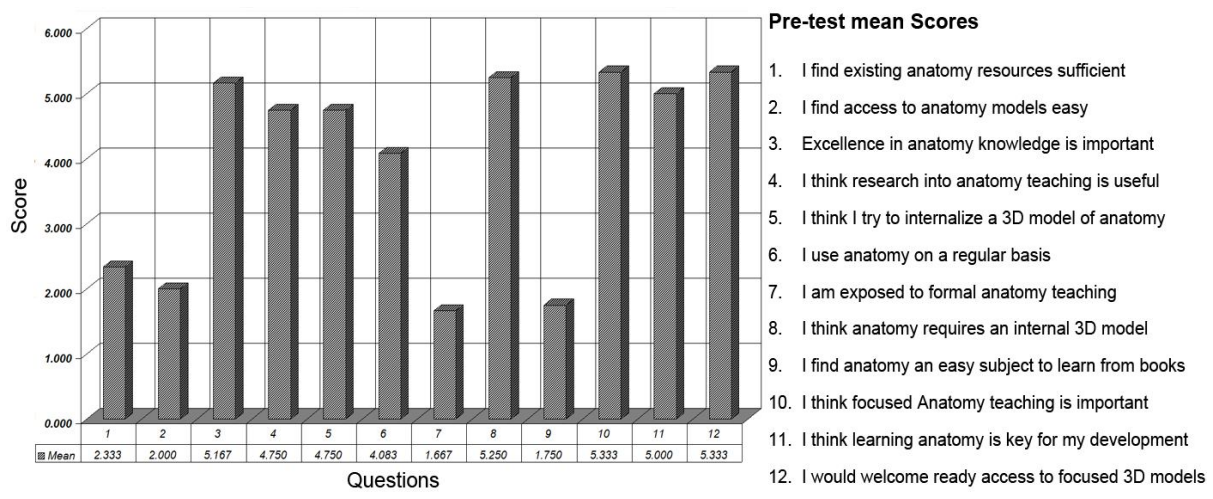

Fig. 5. Pre-test mean scores in all 12 pre-assess Likert questions.

The pre-assessment quiz consisted of open questions relating to the anatomy of the inguinal canal region. It aimed to establish the baseline knowledge of the users prior to any intervention and was compared with the post intervention assessment quiz. The aims of the exercise were two-fold: Identify the user's prior pure anatomy knowledge of the region and furthermore elucidate their ability to comprehend how anatomical structures relate to each other in a $3 \mathrm{D}$ environment. The latter was assessed by questions referring to space relations of the different structures in the $3 \mathrm{D}$ space.

It is of interest to note that overall the pre-intervention scores for both subgroups were disappointingly low, further enhancing the view that anatomy teaching in its current format lacks in its ability to provide in-depth understanding of complex anatomical relationships. Mean scores approached the $50 \%$ mark with no significant differences between subgroups $(\mathrm{p}=0.857)$ and almost negligible variance scores preintervention. Both groups improved on their scores after the teaching intervention, as expected, the traditional method group with a mean improvement in scores of $16 \%$, whilst the VR method group with a mean percentage improvement of $25 \%$. Scores were significantly different between the two groups post intervention $(p=0.041)$ suggesting that users exposed to the VR method accumulated a better understanding 
of the spatial interrelationships of the structural elements of the canal compared to those taught in the traditional method. Furthermore, on further analysis of particular questions relating purely to the spatial relationship of structures, the VR group had an even higher advantage.

On concluding the experiment all users completed the usability questionnaire, where they were asked to grade their views about the educational approach. 25 statements were graded on a 6 point Likert scale ( 1 for strongly disagree to 6 for strongly agree). The views of the two groups were markedly different on almost all points with $\mathrm{p}$ values less than 0.001 on two sample t-testing. Positively phrased statements regarding the educational approach scored very highly in the VR group whilst scoring low in the traditional method group and vice versa for negatively phrased statements, as has been the case in every other case study we have investigated.

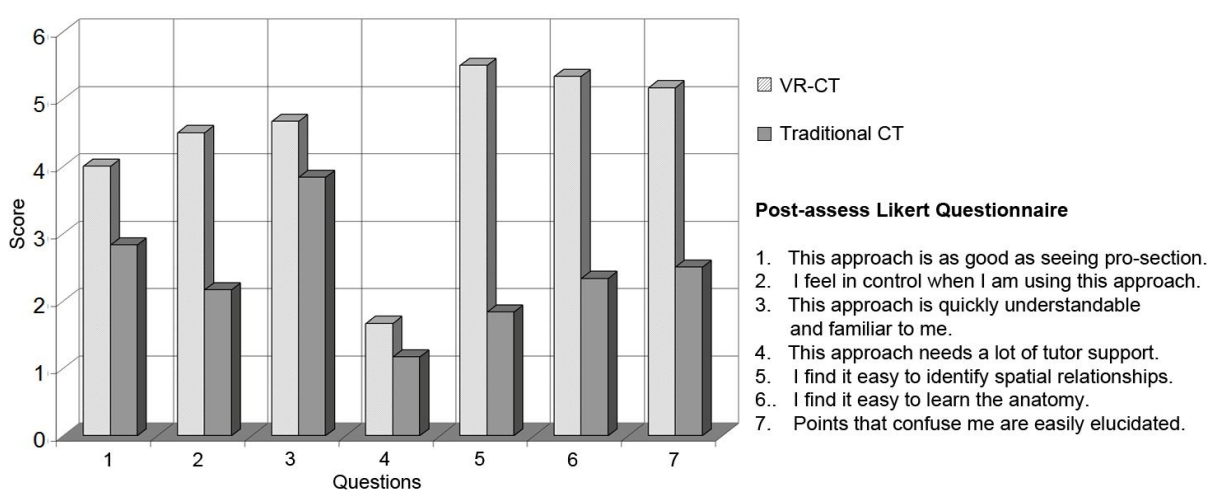

Fig. 6. Post-assess Likert scores in 7 selected questions relevant to the understanding of the $3 \mathrm{D}$ anatomical structures and the need of the tutor support.

The only points that did not exhibit a significant difference related to the ease of use of the system and the familiarity with the interfaces. Remarkably even in these questions where one would have expected the responses to the VR approach to be more guarded, the responses were overall positive, with the majority of users finding the VR system more engaging, interesting and easy to use and more efficient in elucidating spatial inter-relationships of structures. Users preferred the VR system over traditional teaching methods and they were more inclined to recommend it to their peers $(\mathrm{p}<0.001$ compared to the traditional method group responses) as Figure 5 illustrates.

\section{Conclusions}

Overall this paper presented the development process and the challenges that rose during the design and implementation of an augmented reality environment, and the HCI design. A succinct but informative introduction to the visual components of the interface offered a clear understanding of the aims of this work which focused 
primarily in the conveyance of meaningful information to the trainee in subjects that involve complex and convoluted anatomical structures. The suggested interface utilises simplicity to convey as seamlessly as possible the spatial information, facilitates interaction and limits extraneous and unnecessary data while achieving the required fidelity.

A presentation of the educational status quo in medicine offered the background and the reasoning for the development of an augmented reality inguinal canal. To this end it was evident that the present-day trainee remains reliant on inadequate cadaveric exposure, limited two-dimensional resources and inappropriate opportunistic learning through incisional 'windows' in the living patient. In this study we illuminated useful approaches to intuitive design aiming to allow trainees and trainers to maximise learning outcomes. In addition, through the development of appropriate, focused anatomical models we have explored important issues pertaining to the design of practical applications to augment anatomical training which could facilitate in the near future tools also applicable to surgical training in these complex regions.

Evidently the presented outcomes of this preliminary evaluation were in favour of the proposed VR training method in contrast to the traditional method. The evaluation identified weaknesses in the choice of haptic devices. Yet it was strongly suggested by the subjective feedback that there is a place for further research into intuitive interfaces and augmented reality environments that may facilitate the implementation of practical, focused anatomical and potentially surgical training applications.

\section{References}

1. Nicholson D. T., Chalk C., W. Robert, Funnell J., Daniel S. J.: Can Virtual Reality Improve Anatomy Education? A Randomised Controlled Study of a Computer-Generated ThreeDimensional Anatomical Ear Model, Medical Education, Vol 40/11, pp 1081-1087, (2006).

2. Qin Lu and Shuqian Luo: Primary Research of Digital Atlas of Human Anatomy on Virtual Reality, In Proceedinggs of the $2^{\text {nd }}$ International Conference on Bioinformatics and Biomedical Engineering, (ICBBE 08), pp 2442-2445, Shanghai, China, (2008).

3. Turney BW.: Anatomy in a modern medical curriculum", In: Annals of the Royal College of Surgeons of England. 89(2) pp 104-107, (2007).

4. Charissis V., Ward B.M., Naef M., Rowley D., Brady L. and Anderson P.: An Enquiry into VR Interface Design for Medical Training: VR Augmented Anatomy Tutorials for Breast Cancer, in Proceedings of the International Annual Symposium of SPIE, 27-31 January, San Jose, California, USA. (2008).

5. Ornstein M. H. Virtual Reality and Laparoscopic Surgery British Journal of Surgery, Vol 82/6, pp 854-855, (1995)

6. Sang-Hack, Jung \& Bajcsy, R.: Learning Physical Activities in Immersive Virtual Environments. In: IEEE Proceedings of the International Conference on Computer Vision Systems, ICVS '06, St. Johns University, Manhattan, New York City, USA, (2006).

7. Ward B.M., Charissis V., Rowley D., Anderson P., and Brady L.: An Evaluation of Prototype VR Medical Training Environment: Applied Surgical Anatomy Training for Malignant Breast Disease, in Proceedings of the 16th International Conference of Medicine Meets Virtual Reality, Long Beach, California, USA. (2008).

8. Crossan, A., Brewster, S., Reid, S. \& Mellor, D.: Multi-Session VR Medical Training - The HOPS Simulator, in the Proc. of British HCI Conference, London, UK, pp 213-225, Springer, (2002). 